\title{
INERTIAL $h$-COBORDISMS WITH FINITE CYCLIC FUNDAMENTAL GROUP
}

\author{
TERRY C. LAWSON ${ }^{1}$
}

\begin{abstract}
For $M$ a PL $n$-manifold, $n \geqq 5$, let $I(M)$ be the subset of torsions $\sigma \in \mathrm{Wh}\left(\pi_{1} M\right)$ such that the $h$-cobordism $W$ constructed from $M$ with torsion $\sigma$ has its other boundary component PL homeomorphic to $M$. We present three techniques dealing with the determination of $I(M)$ and apply them when $\pi_{1} M=Z_{q}$. We prove: (1) If $n$ is even, $\pi_{1} M \simeq Z_{a}, q$ odd, then $I(M)=$ Wh $\left(\pi_{1} M\right)$. (2) If $n$ is odd, then there exists $M$ with $\pi_{1} M \simeq Z_{q}$ such that $I(M)=\mathrm{Wh}\left(\pi_{1} M\right)$.
\end{abstract}

1. Introduction. We first establish our notation. For convenience we work in the piecewise linear category; similar results hold in the differential and topological categories. $M$ and $N$ will denote closed manifolds of dimension $n \geqq 5$. We use $W$ (and $\left.W^{\prime}\right)$ to denote a compact $(n+1)$ manifold which is an $h$-cobordism between its two boundary components $W_{0}, W_{1}$. " $\simeq$ " will denote "is PL homeomorphic to" or "is (group) isomorphic to", depending on the context. We call an $h$-cobordism $W$ inertial if $W_{0} \simeq W_{1} . I(M)$ will denote the subset of $\mathrm{Wh}\left(\pi_{1} M\right)$ consisting of torsions $\sigma$ (we measure our torsions in the domain space except where indicated otherwise) such that the $h$-cobordism $W(M, \sigma)$ with $W_{0}=M$ and the torsion of $(W, M)$ (i.e. of $M \subseteq W)$ equal to $\sigma$ is inertial; i.e. $W_{1} \simeq M$. Recall that $W(M, \sigma)$ is determined up to a PL homeomorphism which is the identity on $M$ [5].

One of the principal tools of geometric topology is the s-cobordism theorem. Frequently it is used in a context where one needs only to know that a particular $h$-cobordism is inertial. Thus a determination of $I(M)$ becomes of interest. In this note we present three techniques which are relevant to this problem and apply them to the case where $\pi_{1} M \simeq Z_{q}$, the finite cyclic group of order $q$. In this case we show that if $q$ is odd and $n$ is even, then $I(M)=\mathrm{Wh}\left(\pi_{1} M\right)$. If $n$ is odd, the example of lens spaces

Received by the editors September 17, 1973.

AMS (MOS) subject classifications (1970). Primary 57C10, 57D80.

Key words and phrases. Inertial h-cobordism, s-cobordism theorem, Whitehead group, Wall group, pseudo-projective plane.

1 This research was supported in part by a grant from the Tulane University Committee on Research.

(c) American Mathematical Society 1974 
shows that $I(M)$ may be 0 [5]. We show that if $n$ odd, $q$ arbitrary, then there is a manifold $M$ with $\pi_{1} M \simeq Z_{q}$ such that $I(M)=\mathrm{Wh}\left(\pi_{1} M\right)$.

2. Constructions and applications. Our first technique involves an application of surgery theory; for further details on surgery, see [7]. Our terminology will follow [7]. Let $S_{\mathrm{PL}}(M)$ denote the (simple) homotopy triangulations of $M$. This has a distinguished element $\left[1_{M}\right]=[f]$, where $f: M^{\prime} \rightarrow M$ is a simple homotopy equivalence that is homotopic to a PL homeomorphism. Let

$$
H^{m}(\pi)=\left\{\tau \in \mathrm{Wh}(\pi): \tau=(-1)^{m} \bar{\tau}\right\} /\left\{\tau+(-1)^{m} \bar{\tau}\right\},
$$

where $\bar{\tau}$ denotes the conjugate of $\tau$. Rothenburg (cf. [7]) has exhibited an exact sequence, three terms of which are

$$
H^{n+2}(\pi) \stackrel{s}{\longrightarrow} L_{n+1}^{s}(\pi) \stackrel{t}{\longrightarrow} L_{n+1}^{h}(\pi) .
$$

Wall [7, p. 108] describes an action of $L_{n+1}^{s}\left(\pi_{1} M\right)$ on $S_{\mathrm{PL}}(M)$, which we denote by $\cdot$. In particular, the image of $H^{n+2}\left(\pi_{1} M\right)$ under $s$ acts on $\left[1_{M}\right] \in S_{\mathrm{PL}}(M)$ as follows. If $\sigma \in \mathrm{Wh}\left(\pi_{1} M\right)$ satisfies $\sigma=(-1)^{n} \bar{\sigma}$, then $\sigma$ represents an element $[\sigma]$ of $H^{n+2}\left(\pi_{1} M\right) ; s([\sigma]) \cdot\left[1_{M}\right]$ is represented by $r i: W_{1} \rightarrow W_{0}=M$, where $W=W(M, \sigma), i: W_{1} \rightarrow W$ is the inclusion and $r: W \rightarrow W_{0}$ is a deformation retraction. The condition $\sigma=(-1)^{n} \bar{\sigma}$ is required for $r i$ to be a simple homotopy equivalence. If $s([\sigma]) \cdot\left[1_{M}\right]=$ $\left[1_{M}\right]$, then $r i$ is homotopic to a PL homeomorphism and $W_{1} \simeq M$. In general; determining this action is a highly nontrivial problem; however, the difficulties collapse under appropriate algebraic assumptions.

Proposition 1. Let $n \geqq 5$ be even and suppose conjugation is trivial in $\mathrm{Wh}\left(\pi_{1} M\right)$. Assume also that the map $t: L_{n+1}^{s}\left(\pi_{1} M\right) \rightarrow L_{n+1}^{n}\left(\pi_{1} M\right)$ is injective. Then $I(M)=\mathrm{Wh}\left(\pi_{1} M\right)$.

Proof. Since $n$ is even and conjugation is trivial, each $\sigma \in \mathrm{Wh}\left(\pi_{1} M\right)$ satisfies $\sigma=(-1)^{n} \bar{\sigma}$. If $s([\sigma]) \cdot\left[1_{M}\right]=\left[1_{M}\right]$, then $\sigma \in I(M)$. But im $s=$ ker $t=0$.

Corollary 1. If $n \geqq 5$ is even and $\pi_{1} M \simeq Z_{q}, q$ odd, then $I(M)=$ $\mathrm{Wh}\left(\pi_{1} M\right)$.

Proof. Conjugation is trivial in $\operatorname{Wh}\left(Z_{a}\right)$ [2]. A. Bak [1] has shown that $L_{n+1}^{s}(\pi)=0=L_{n+1}^{h}(\pi)$ for $\pi$ a finite abelian group of odd order, hence for $\pi=Z_{q}$.

Remarks. 1. The ideas behind Proposition 1 have been known for some time. What was needed to apply them were computations of Wall groups such as Bak's. 
2. Note that Corollary 1 is nontrivial since $\mathrm{Wh}\left(Z_{q}\right)$ is a direct sum of $\phi(q) / 2-1$ copies of $Z, q \geqq 3$, where $\phi$ is the Euler $\phi$-function [2].

3. $2 \mathrm{Wh}\left(\pi_{1} M\right)=\left\{\sigma \in \mathrm{Wh}\left(\pi_{1} M\right): \sigma=2 \tau\right\}$ is easily seen to lie in $I(M)$ via the doubling construction of Milnor [5] when conjugation is trivial. In this case $H^{n+2}\left(\pi_{1} M\right)=\mathrm{Wh}\left(\pi_{1} M\right) / 2 \mathrm{Wh}\left(\pi_{1} M\right)$.

4. Let $\pi_{1} M$ be a finite abelian group of odd order, $C=\left\{\sigma \in \mathrm{Wh}\left(\pi_{1} M\right)\right.$ : $\sigma=\bar{\sigma}\}$. Then the proof of Proposition 1 shows that $C \subset I(M)$.

When $n$ is odd, the technique above appears to be more difficult to apply; it is useless for $\pi_{1} M \simeq Z_{q}$. A relevant question for $n$ odd is one of realizability: Given $\sigma \in \mathrm{Wh}(\pi)$, does there exist a manifold $M$ with $\pi_{1} M=\pi$ and $\sigma \in I(M)$ ? In particular, can one find $M$ such that $\mathrm{Wh}\left(\pi_{1} M\right)=$ $I(M)$ ? The Milnor doubling construction yields no examples when $\sigma=\bar{\sigma}$, as in $\mathrm{Wh}\left(\boldsymbol{Z}_{q}\right)$. However, a minor modification of it (which is also related to Farrell's fibering theorem [3]) does lead to some results.

Suppose $W$ is an $h$-cobordism with $W_{0}=M, W_{1}=N$ and $W^{\prime}$ is an $h$ cobordism with $W_{0}^{\prime}=N$ and $W_{1}^{\prime} \simeq M$. Suppose $f: N \rightarrow N$ is a PL homeomorphism. Let $\sigma, \sigma^{\prime} \in \mathrm{Wh}\left(\pi_{1} N\right)$ be the torsions of $(W, M),\left(W^{\prime}, N\right)$ as measured in $\mathrm{Wh}\left(\pi_{1} N\right)$. That is, if $\sigma_{1}$ and $\sigma_{1}^{\prime}$ denote the torsions of $(W, M)$ and $\left(W^{\prime}, N\right)$ measured in $\mathrm{Wh}\left(\pi_{1} W\right)$ and $\mathrm{Wh}\left(\pi_{1} W^{\prime}\right)$, respectively, and $r_{N}: W \rightarrow N, \quad r_{N}^{\prime}: W^{\prime} \rightarrow N$ are deformation retractions, then $\left(r_{N}\right)_{\#} \sigma_{1}=\sigma$ and $\left(r_{N}^{\prime}\right)_{\#} \sigma_{1}^{\prime}=\sigma^{\prime}$; it turns out to be technically simpler to work in Wh $\left(\pi_{1} N\right)$. Then the torsion of $\left(W \cup_{f} W^{\prime}, M\right)$ as measured in $\mathrm{Wh}\left(\pi_{1} N\right)$ is $\sigma+f_{\#} \sigma^{\prime}$. Two special cases of interest are where $W^{\prime}=W$ (inverted so $W_{0}^{\prime}=W_{1}$, $\left.W_{1}^{\prime}=W_{0}\right)$ and where $W^{\prime}$ is the inverse of $W$, i.e. $W \cup W^{\prime} \simeq M \times 1$. In the first case $\sigma^{\prime}=-\bar{\sigma}$; in the second case $\sigma^{\prime}=-\sigma$. Thus $\left(r_{M} i_{N}\right)_{\#}\left(\sigma-f_{\#} \bar{\sigma}\right)$ and $\left(r_{M} i_{N}\right)_{\#}\left(\sigma-f_{\#} \sigma\right)$ are in $I(M)$ where $i_{N}: N \rightarrow W$ is the inclusion and $r_{M}: W \rightarrow M$ is a deformation retraction. If $\sigma=\bar{\sigma}$, the two cases coincide. The first case is merely a slight modification of the doubling construction. The second case arises naturally in trying to fiber $N_{f}=N \times[0,1] /(x, 1) \sim$ $(f(x), 0)$ over the circle when one splits along $M$. For this construction to be of any value, there must exist manifolds $N$ and PL homeomorphisms $f$ of $N$ which induce nontrivial maps $f_{*}$ of $\pi_{1} N$ and then $f_{\#}$ of $\mathrm{Wh}\left(\pi_{1} N\right)$. Our next proposition concerns $f_{*}$ when $\pi_{1} N=Z_{q}$; we only examine $f_{\#}$ for the special case of $Z_{5}$.

Proposition 2. Let $\alpha_{r}$ be the automorphism of $Z_{q}$ such that $\alpha_{r}(1)=r$, $(r, q)=1$. Then there is a manifold $N$ (of any given dimension $\geqq 5$ ) and $a$ PL homeomorphism $f: N 与$ with $\pi_{1} N=Z_{q}$ and $f_{*}=\alpha_{r}$.

Proof. Let $P_{q}$ denote the pseudoprojective plane $S^{1} \cup_{q} e^{2}$. Olum [6] proves that there is a simple homotopy equivalence $g: P_{Q} \subseteq$ with $g_{*}=\alpha_{r}$ (under the natural isomorphism $\pi_{1} P_{q} \simeq Z_{q}$ ). Embed $P_{q}$ as a subcomplex of $E^{n+1}, n \geqq 6$ and let $N$ be the boundary of a regular neighborhood $R$ of $P_{q}$. 
The composition of $g$ with the inclusion of $P_{q}$ into the interior of $R$ is homotopic to an imbedding $h$. By [4] and uniqueness of regular neighborhoods, $h$ may be extended to an imbedding $k$ of $R$ into the interior of $R$. Since $g$ is a simple homotopy equivalence, so is $k$; excision and the $s$-cobordism theorem then imply that $R \backslash$ int $k(R) \simeq N \times[0,1]$. Using collars, we may modify $k$ to give a PL homeomorphism $l: R \rightarrow R$. Letting $f=l \mid N$, then $f_{*}=\alpha_{r}$ (in terms of the isomorphisms $\pi_{1} N \simeq \pi_{1} R \simeq \pi_{1} P_{q} \simeq Z_{q}$ ).

Thus we may realize any possible automorphism of $\boldsymbol{Z}_{q}$. The next question is whether $\left(\alpha_{r}\right)_{\#}: \mathrm{Wh}\left(\boldsymbol{Z}_{q}\right) \subseteq$ is ever nontrivial. Since we have a more effective technique for $Z_{q}$, we content ourselves with one example: $q=5$. Then $\operatorname{Wh}\left(Z_{5}\right) \simeq Z$, generated by the unit $t+t^{-1}-1 \in Z\left(Z_{5}\right)$, with inverse $t^{2}+t^{-2}-1$, where $t$ denotes the generator of $Z_{5}$ [5]. Thus the automorphism $\alpha_{2}: Z_{5} \lesseqgtr$ has $\left(\alpha_{2}\right)_{\#}\left(t+t^{-1}-1\right)=t^{2}+t^{-2}-1=\left(t+t^{-1}-1\right)^{-1}$. Thus $2 \mathrm{Wh}\left(Z_{5}\right) \subset$ $I(M)$, where $M=W_{1}\left(N,\left[t+t^{-1}-1\right]\right), N$ as in Proposition 2.

The proof of Proposition 2 suggests another technique which yields our realizability theorem for $Z_{Q}$ using another result of Olum [6].

Proposition 3. Let $n \geqq 5, X \subset E^{n+1}$ a 2-complex and $g: X \hookrightarrow$ a homotopy equivalence with torsion $\tau \in \mathrm{Wh}\left(\pi_{1} X\right)$. Then if $N$ is the boundary of a regular neighborhood $R$ of $X$, we have $F_{\#} \tau \in I(N)$ where $F$ is the composition of isomorphisms $\pi_{1} X \simeq \pi_{1} R \simeq \pi_{1} N$ induced by inclusions.

Proof. Homotope $X \rightarrow^{g} X \varsigma$ int $R$ to an imbedding $h . h$ then extends to an imbedding $k: R \rightarrow$ int $R$ by [4]. Then $R \mid$ int $k(R)$ is an inertial $h$ cobordism with boundary $N \cup k(N)$. Using excision, one computes the torsion of $k(N) \subset R \mid$ int $k(R)$ as $G_{*} \tau \in \mathrm{Wh}\left(\pi_{1}(k(N))\right)$ where $G: \pi_{1} X \rightarrow$ $\pi_{1} k(N)$ is the composition $\pi_{1} X \rightarrow^{g}{ }^{*} \pi_{1} X \rightarrow^{i}{ }^{*} \pi_{1} k(R) \rightarrow^{j_{*}-1} \pi_{1} k(N)$. Transferring from $k(N)$ to $N$ via $(k \mid N)^{-1}$ gives $F_{\#} \tau \in I(N)$ for $F=(k \mid N)_{*}^{-1} G$. One then checks $F$ is just the composition of isomorphisms $\pi_{1} X \simeq \pi_{1} R \simeq$ $\pi_{1} N$ induced by inclusions.

Corollary 2. Given $n \geqq 5, q \in N$, there is a manifold $N$ of dimension $n$ with $\pi_{1} N \simeq Z_{q}$ and $\mathrm{Wh}\left(\pi_{1} N\right)=I(N)$.

Proof. This follows from Proposition 3 with $X=P_{q}$ using Olum's result [6] that there are self homotopy equivalences of $P_{q}$ with prescribed torsion in $\mathrm{Wh}\left(\pi_{1} P_{q}\right)$.

Remark. Propositions 2 and 3 make it clear that the realizability problem is really one of $\mathrm{CW}$ complexes and (simple) homotopy equivalences.

\section{REFERENCES}

1. A. Bak and W. Scharlau, Witt groups of orders and finite groups, 1972 (preprint).

2. H. Bass, Algebraic K-theory, Benjamin, New York, 1968. MR 40 \#2736. 
3. F. T. Farrell, The obstruction to fibering a manifold over a circle, Bull. Amer. Math. Soc. 73 (1967), 737-740. MR 35 \#6151.

4. V. K. A. M. Gugenheim, Some theorems on piecewise linear embedding, Proc. Nat. Acad. Sci. U.S.A. 38 (1952), 333-337. MR 14, 74.

5. J. Milnor, Whitehead torsion, Bull. Amer. Math. Soc. 72 (1966), 358-426. MR 33 \#4922.

6. P. Olum, Self-equivalences of pseudo-projective planes. II: Simple equivalences, Topology 10 (1971), 257-260. MR 43 \#1185.

7. C. T. C. Wall, Surgery on compact manifolds, Academic Press, New York, 1971.

Department of Mathematics, Tulane University, New Orleans, Louisiana 70118 